\title{
A DEMONSTRATION OF THE SITE OF ACTION OF INTRAVENOUS LIGNOCAINE
}

\author{
S. A. Flemnn, B.A., M.D., F.F.A.R.C.S., J. A. VEiga-Ptres, M.D., \\ C.H.D., D.M.R.D., D.R.D., R. M. MCCUTCHEON, M.D., AND \\ C. I. EManuel, M.B., B.s.
}

\section{INTRODUCTION}

The INTRAVENous INJECTION of lignocaine distal to a tourniquet has been found a useful, time-saving, safe method of anaesthesia for short operations on a limb. Since the re-introduction of this technique two years ago, there has been some controversy concerning the site of action of the agent. Sorbie and Chacha ${ }^{1}$ felt that analgesia was produced by an effect on the main nerve trunks. Our observation of over 2,000 cases has led us to doubt this conclusion. The analgesia may be spotty, with one area well anaesthetized while an adjacent area, supplied by the same nerve, has full appreciation of pin-prick. This does not support the idea that the main nerve trunks are the principle site of action of the anaesthetic, but rather suggests that the drug acts at the tissue level on the peripheral nerve endings.

Intravenous regional anaesthesia of the arm was induced with lignocaine in three members of the Department of Anaesthesia of the University of Lagos Teaching Hospital. Site of cuff, volume and concentration of solution, direction of the needle, and venous valves have all been mentioned as factors associated with the rapidity of onset, extent, and intensity of analgesia produced. One member of the staff experienced immediate and complete anaesthesia following each injection, whereas the other two subjects required up to several minutes and larger volumes to produce similar analgesia. One of the latter, in fact, never had complete analgesia of the fingers even when the cuff was positioned at the wrist and 20 c.c. of one per cent lignocaine were injected through a needle directed distally. Another interesting feature was the rare observation of ringing in the ears and changes in sensorium while the injection was being made, even though the blood-pressure cuff was fully inflated to our usual pressure level of 250-300 mm. Hg. It was thought that the solution might pass through medullary veins in the bone.

To demonstrate the distribution of the injected lignocaine, Hypaque was added to the solution, and serial $\mathrm{X}$-rays were taken.

\section{METHOD}

Four trials were undertaken using 10 c.c. of 85 per cent Hypaque well mixed with the solution of lignocaine. The upper limit of lignocaine dosage was $200 \mathrm{mg}$. In each experiment the arm was elevated for 60 seconds before the cuff was

-Departments of Anaesthesia and Radiodiagnosis, University of Lagos Medical School, Lagos, Nigeria.

Can. Anaes. Soc. J., vol. 13, no. 1, January, 1966 
inflated. The injection was completed in one to two minutes except in experiment two. X-rays were taken at ten-second intervals during the first two minutes, and at two-minute intervals thereafter. The amount of solution injected and the areas of analgesia (response to pin-prick) were noted with each $\mathrm{X}$-ray exposure. The cuff was left on the arm for ten minutes after injection to allow fixation of lignocaine in the tissues to prevent toxic manifestations on its release.

\section{Observations}

The onset of analgesia compared closely with previous injections of one per cent lignocaine without Hypaque in the same individuals, but there was uncomfortable burning associated with the injection of the Hypaque solutions.

Correlation of the area of anaesthesia with the X-ray position of the contrast medium was possible with the co-operation of the radiologist.

\section{Experiment 1}

Twenty c.c. of one per cent lignocaine with Hypaque solution was injected into the forearm, with the cuff above the elbow. Good filling of veins was seen in the forearm, and ultimately some on the dorsum of the hand, while analgesia spread from the back to the front of the forearm to the dorsum of the hand. There was no contrast medium seen in the fingers, and there was no anaesthesia of the fingers (Fig. 1). It is interesting to recall that the radial nerve supplies both the back of the hand and the dorsum of the fingers.

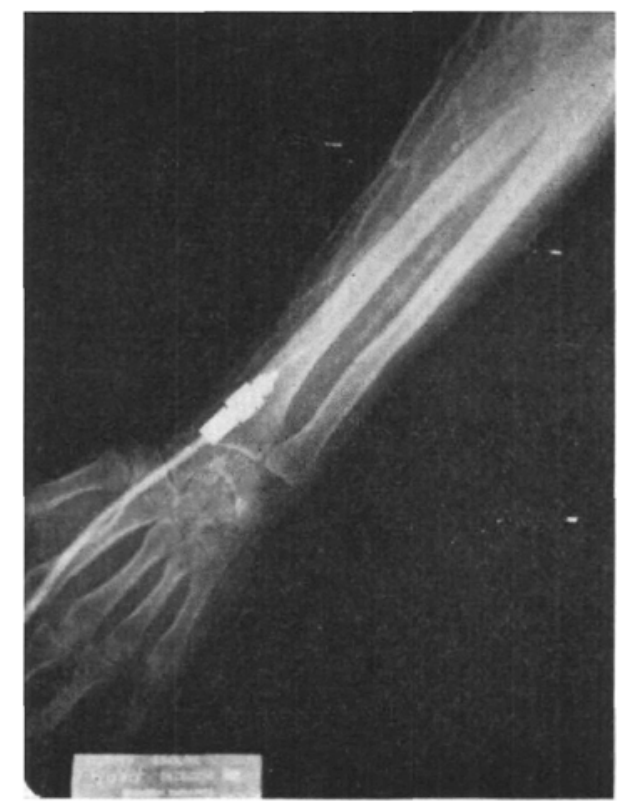

Ficure 1. No contrast medium seen in the fingers. No anaesthesia of the fingers. 


\section{Experiment 2}

With the cuff above the elbow, 40 c.c. of 0.5 per cent lignocaine with Hypaque solution was injected into a vein on the dorsum of the hand through a needle directed distally. Good filling of the dorsum of the hand occurred just to the first set of valves, about one inch above the metacarpophalangeal joint (Fig. 2). Filling then moved cephalad up the arm to the cuff, with analgesia corresponding to the areas in which contrast medium was observed. After the forearm was filled by approximately 20 c.c. in two minutes, the solution escaped distally past the venous valves to a point just distal to the metacarpophalangeal joint in each finger, where again a line of valves was outlined (Figs. 3 and 4). With further injection of 5 c.c. of solution, the contrast medium finally reached the digital veins, and it was then that the onset of anaesthesia was noted in the fingers (Fig. 5). At this stage a soft tissue blush was observed, deepening as the analgesia became complete (Fig. 6 after 35 c.c. at $9 \%$ minutes and Fig. 7 after 40 c.c. at 13 minutes).

\section{Experiment 3}

With 20 c.c. of one per cent lignocaine with Hypaque solution, the needle directed distally in the dorsum of the hand, and the cuff just above the wrist, the valves were again outlined nicely. The thumb was rapidly perfused with the contrast material and analgesia was noted first in the thumb and dorsum of hand, then in the palmar area, and finally in the fingers, except for the dorsum

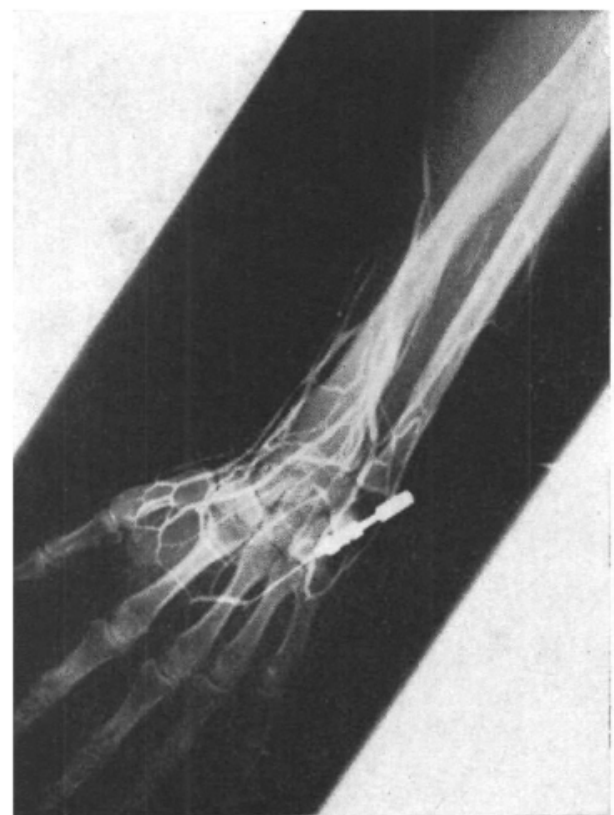

Freure 2. Solution impeded by venous valves.

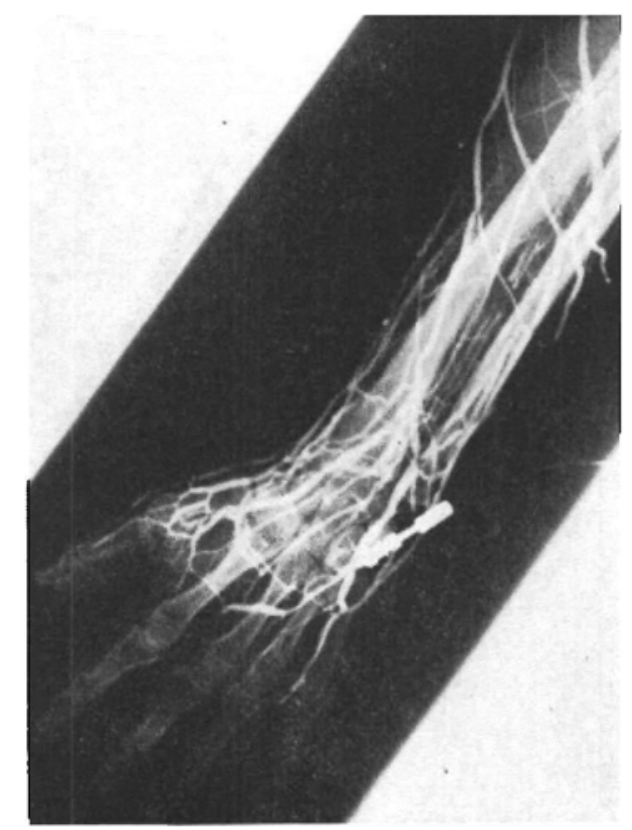

Figure 3. Blush of contrast medium seen over carpal area accompanied by anaesthesia. 


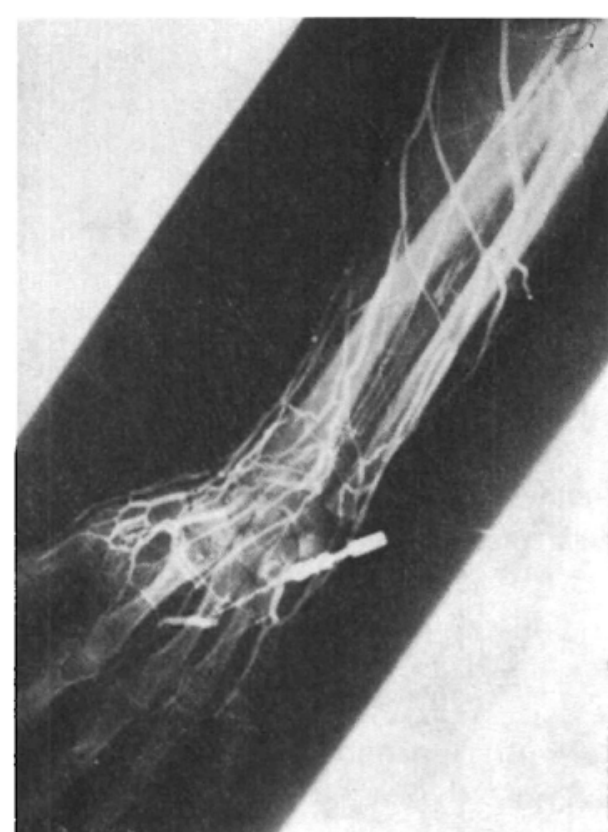

FIGUre 4. Solution reaches distal vessels.

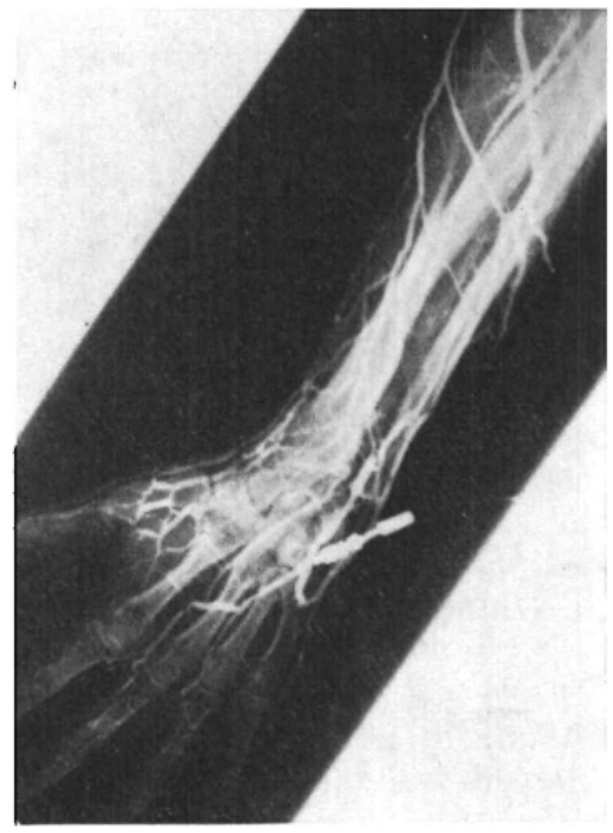

Ficure 5. Deeper and more extensive blush. Onset of anaesthesia of fingers.

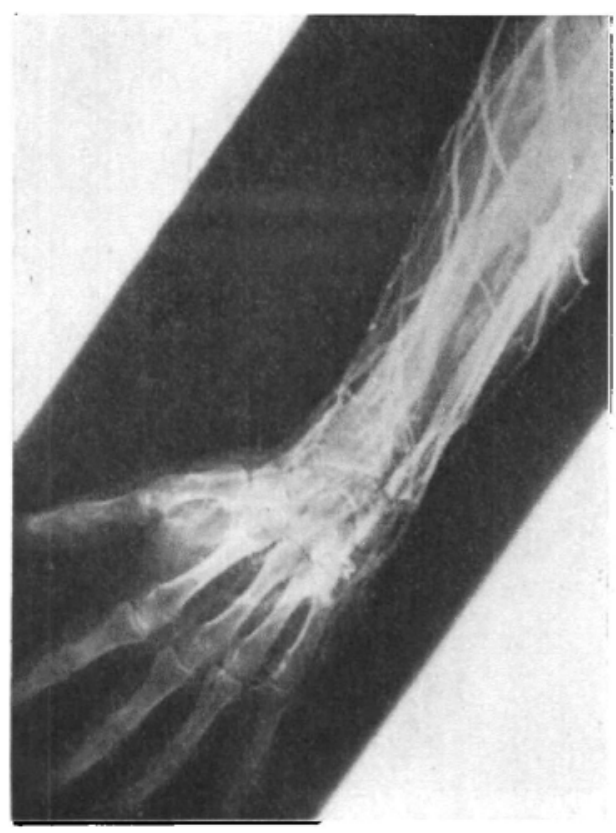

Figure 6. Complete anaesthesia of hand.

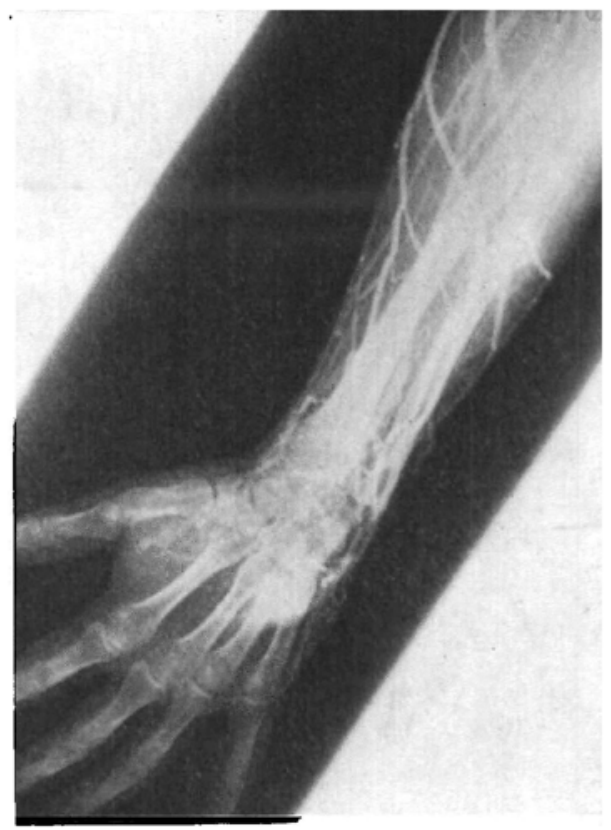

Figure 7. Blush. 
of the middle phalanges of the fourth and fifth, which were anaesthetized after four minutes. It was interesting to observe contrast medium passing under the inflated cuff and through veins above the cuff after 15 c.c. had been injected (1\% minutes, Fig. 8). The veins between the valves and the cuff were greatly distended with contrast material. The blush in the tissues and the drainage of the contrast medium after the cuff was deflated are apparent in Figure 9.

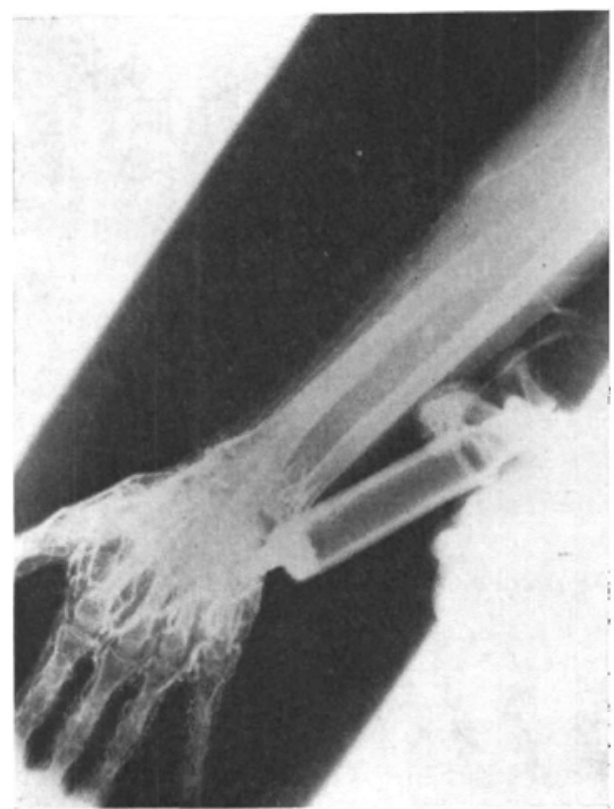

Ficune 8. Cuff above wrist inflated; escape of solution past cuff. Fifteen c.c. of solution injected in $1 \frac{1}{2} \mathrm{~min}$.

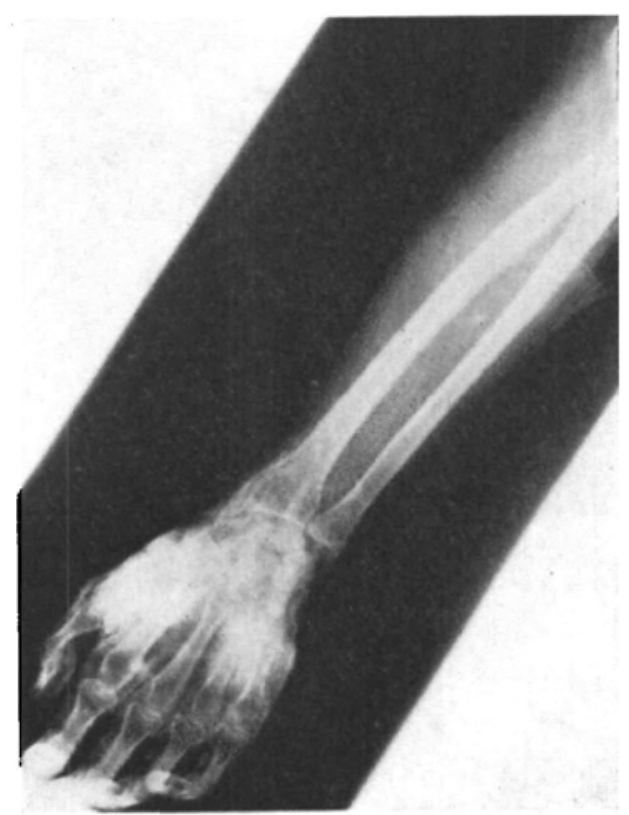

Ficune 9. Cuff deflated after ten minutes.

\section{Experiment 4}

In one individual, after injection of 15 c.c. of lignocaine by the method used in Experiment 3, the valves did not stand out as in the previous two subjects. The contrast material passed into the fingers within 20 seconds, but none was seen passing under the cuff. Anaesthesia developed within 30 seconds, before the injection was completed, and a soft tissue blush developed at this time, much sooner than in the previous cases (Figs. 10 and 11).

The quantity of solution played an important part in producing complete anaesthesia. The direction of injection may play a minor role.

An interesting feature seen in these cases was the part played by the venous valves in the hand and fingers. In one subject, the valves appeared to be overwhelmed very easily, contrast medium passed immediately into the fingers, the soft tissue blush and anaesthetic both appeared early, and no contrast material was squeezed past the inflated cuff. In the hand in Experiments 1 and 3 (same 


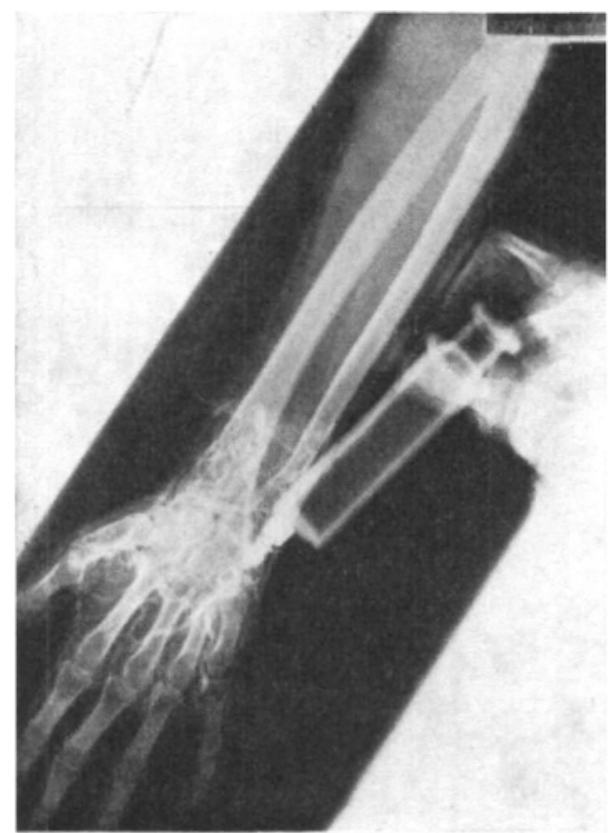

Figure 10. No leak past cuff. Few valves apparent.

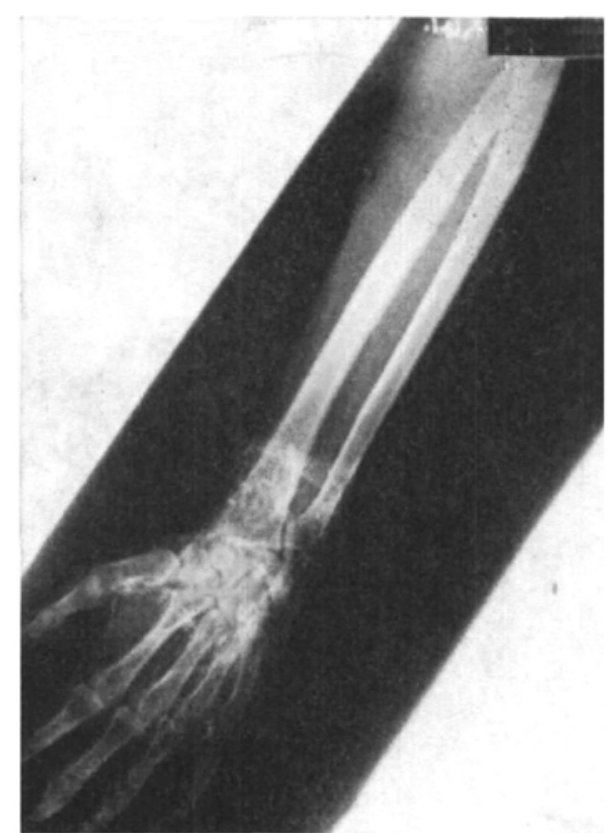

Frgure 11. One minute after injection. Complete anaesthesia.

person), valves clearly prevented passage of the Hypaque into the fingers until enough solution had been injected to cause sufficient pressure to overcome them and to force the solution into the digital veins. In fact, the valves in the hand in this case were so competent that contrast medium was forced past the cuff inflated to over $250 \mathrm{~mm}$. Hg. It is in this type of patient that 40 c.c. of solution would probably not be sufficient to give digital anaesthesia with the cuff at the elbow, and it is easily seen why cerebral "sensations" may occur during the injection of the solution.

Caution should be exercised when injecting radio-opaque solutions in such experiments as these, as a local reaction to the Hypaque was experienced after a second injection during this investigation.

\section{Conclusion}

It would seem to be conclusively shown that intravenous lignocaine injected distal to a tourniquet acts at the tissue level on the nerve endings. The onset of analgesia and the distribution of contrast material in any given region correlated perfectly. In each instance analgesia occurred when and where the soft tissue blush indicated tissue perfusion by the lignocaine-Hypaque solution. Where there was no contrast medium, there was no anaesthesia. This would explain the clinical observation of areas of analgesia and complete preservation of sensation existing in the distribution of the same nerve. 


\section{SUMMARY}

The intravenous injection of lignocaine distal to a tourniquet has been found a useful, time-saving, safe method of anaesthesia for short operations on a limb. There has been some controversy concerning the site of action of the agent.

A solution of lignocaine containing Hypaque as a radio-opaque medium was used to permit radiological demonstration of the distribution of the solution injected into the veins of the arm distal to the tourniquet. X-ray pictures of the injected limb were correlated with the area of anaesthesia existing at the time of the X-ray exposure, and it was shown that anaesthesia existed only in those areas where the radio-opaque medium was demonstrated actually in the tissues. Anaesthesia spread as the radio-opaque material spread into further tissue. It would seem to be conclusively shown that intravenous lignocaine injected distal to a tourniquet acts at the tissue level on the nerve endings. This would explain the clinical observation of areas of analgesia and complete preservation of sensation existing in the distribution of the same nerve.

\section{RésuMÉ}

L'injection intraveineuse de lignocaïne dans la partie distale d'un membre par rapport au garrot s'est avérée utile. Elle sauve du temps et, pour des opérations de courte durée aux membres, elle est une méthode d'anesthésie de toute sécurité. Il existe tout de même du désaccord sur le site d'action de l'agent.

Nous avons employé une solution de lignocaine contenant de l'hypaque comme agent radio-opaque pour réaliser une démonstration radiologique de la distribution de la solution injectée dans les veines d'un bras au-dessous d'un garrot. Les radiographies du membre injecté correspondaient à l'étendue de l’anesthésie qui existait au moment de prendre le cliché et il a été démontré que l'anesthésie n'existait qu'aux endroits où la présence de l'agent radio-opaque a été trouvé dans les tissus par la radiographie. L'anesthésie s'étend tout comme l'agent radio-opaque dans des tissus plus éloignés. Il semble bien que cela démontre, en définitive, que la lignocaine, en injection endoveineuse au-dessous d'un tourniquet, agit au niveau des tissus sur les terminaisons nerveuses. Cela expliquerait l'observation clinique de zones d'analgésie et de complète conservation de la sensibilité existant dans la distribution du même nerf.

\section{ACKNOWLEDGMENTS}

We wish to acknowledge the interest of Professor Wheeler Haines and Mr. E. A. Okpa, in the Department of Anatomy, who helped in the discussion and direction of these investigations.

\section{REFERENCES}

1. Sorbie, C. \& Chacha, P. B. Regional Anaesthesia by the Intravenous Route. Brit. Med. J., 1: 957 (1985). 